\title{
Artritis séptica
}

\section{septic arthritis}

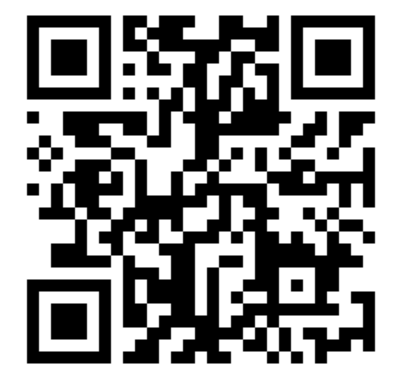

Recibido 05/06/2021
1Dra. Gindreska Paizano Vanega Hospital Jerusalén, San José, Costa Rica

(10) https://orcid.org/0000-0002-9265-820X

${ }^{2}$ Dr. Alexander Araya Oviedo Hospital del Trauma, San José, Costa Rica (iD) https://orcid.org/0000-0003-2641-380X

${ }^{3} \mathrm{Dr}$. Minor Stuard Chacón Díaz Área de Salud de Tilarán. San José, Costa Rica

(D) https://orcid.org/0000-0002-2213-5411

Corregido $10 / 06 / 2021$
Aceptado 20/06/2021

\section{RESUMEN}

La artritis séptica es una patología infecciosa producida por la colonización de un microorganismo piógeno en la cavidad articular, produce una rápida destrucción el cual condiciona el deterioro de la función articular y una notable morbimortalidad, obligando a considerarla una verdadera urgencia médica. Los hallazgos clínicos que deben hacer considerar la posibilidad diagnóstica son: el dolor, la tumefacción o la impotencia funcional. Las pruebas de laboratorio y de imagen ayudan a confirmar el diagnóstico. La artrocentesis juega un papel clave en el diagnóstico y el tratamiento de la artritis séptica, por lo que debe realizarse lo antes posible. El tratamiento se debe iniciar precozmente con el fin de evitar secuelas, basándose en la antibioterapia junto con el drenaje quirúrgico.

PALABRAS CLAVE: artritis séptica; osteomielitis; artrocentesis; análisis de química sinovial.

\section{ABSTRACT}

Septic arthritis is an infectious pathology produced by the colonization of a pyogenic microorganism in the articular cavity, producing a rapid destruction which conditions the deterioration of the articular function and a remarkable morbimortality, forcing to consider it as a real medical emergency. The clinical findings that should lead to consider the possibility of diagnosis are pain, swelling or functional impotence. Laboratory and imaging tests help to confirm the diagnosis. Arthrocentesis plays a key role in the diagnosis and treatment of septic arthritis and should be performed as soon as possible. Treatment should be initiated early in order to avoid sequelae, based on antibiotherapy together with surgical drainage. 
KEYWORDS: septic arthritis; osteomyelitis; arthrocentesis; synovial chemistry analysis.

${ }^{1}$ Médica general, graduada de la Universidad Latina de Costa Rica (U. Latina). Cód. MED13470. Correo: ginpv@hotmail.com

${ }^{2}$ Médico general, graduado de la Universidad Internacional de las Américas (UIA). Cód. MED13605. Correo: alexarao89@hotmail.com

${ }^{3}$ Médico general, graduado de la Universidad de Ciencias Médicas. Cód. MED16659.

\section{INTRODUCCIÓN}

La artritis séptica (AS) es una enfermedad, que en la mayoría de las ocasiones resulta en la afección funcional de la articulación de carácter irreversible. Se define como la infección aguda de la membrana sinovial causada por agentes infecciosos que provocan el deterioro del cartílago articular (1). Más del $90 \%$ son monoarticulares, siendo las articulaciones de miembros inferiores las más frecuentemente afectadas (2.3). Esta patología afecta varias articulaciones principalmente la rodilla, seguida de la cadera, los hombros, muñecas y codos. En pacientes adultos usuarios de drogas intravenosas se ven más afectadas las articulaciones esternoclaviculares y la sacroiliaca $(4,5)$.

Hasta en un $30 \%$ de niños (sobre todo, neonatos y lactantes) coexisten osteomielitis y AS especialmente en las articulaciones de hombro y cadera por la existencia de metáfisis intraarticular. El paciente requiere una atención preferente, ya que la demora en el tratamiento puede conducir a la afectación irreversible de la epífisis (5).

Su presentación clínica es por lo general aguda, traducida en síntomas locales como dolor intenso de la articulación, disminución de la movilidad articular, aumento de temperatura local e imposibilidad para la marcha, fiebre, compromiso del estado general (6).

El diagnóstico temprano evita complicaciones y está basado en elementos clínicos, imagenológicos y microbiológicos. La estrategia de tratamiento debe ser agresiva y enfocada según el posible agente causal de la enfermedad $(7,8)$.
Se realiza una revisión sistema que permite valorar la bibliografía más reciente de AS, con el objetivo de profundizar y actualizar a los profesionales de salud, sobre la importancia de identificar las características clínicas y realizar un diagnóstico temprano, para implementar el tratamiento más adecuado en dicha patología.

\section{MÉTODO}

Se realiza una revisión de artículos y de estudios científicos relevantes, que den validez a lo planteado en el objeto de esta investigación y como criterio de inclusión, aquellos artículos publicados entre los años 2014-2020, en idiomas: inglés y español; tales como Medical Literature Analysis and Retrieval System Online (MEDLINE/PUBMED), Cochrane Database (COCHRANE DATABASE), Literatura Latino-Americana y del Caribe en Ciencias de la Salud (LILACS/BIREME), Cumulative Index to Nursing and Allied Health Literature (CINAHL), Web of Science (WEB OF SCIENCE) y Scientific Electronic Library Online (SciELO).

\section{EPIDEMIOLOGÍA}

La incidencia estimada de AS en países industrializados ha sido establecida en 2-6 casos por 100,000 habitantes/año, es más frecuente en varones con proporciones que oscilan entre el 55 y $60 \%$; el grupo de edad que parece afectarse con más frecuencia en los casos de AS de rodilla es entre 45-65 años (9).

Para que se desarrolle una AS existen una serie de factores de riesgo en adultos y en 
niños que se describen a continuación (10). En población pediátrica la incidencia anual es de alrededor de 4:100.000 niños; siendo el género masculino más propenso, las articulaciones más frecuentemente afectadas son la cadera, rodilla y tobillo (11). Pese al desarrollo de nuevos antibióticos y a la disponibilidad de eficaces medios de diagnóstico, las artritis sépticas constituyen todavía un problema importante, muchos de cuyos aspectos epidemiológicos no se conocen con certeza, y probablemente, varían según el área sanitaria (12).

La AS de rodilla después de una reconstrucción del ligamento cruzado anterior es una complicación poco frecuente pero grave, con una incidencia reportada en la literatura del $0.14 \%$ al $1.7 \%$. Incluso con el mejor tratamiento, existe un riesgo de daño articular, de pérdida del injerto, riesgo de disfunción articular a largo plazo por laxitud ligamentosa, artritis postinfecciosa o artrofibrosis. (13).

\section{ETIOLOGÍA}

Desde el punto de vista microbiológico la infección hematógena es la vía más frecuente en todas las edades y casi cualquier bacteria es capaz de causas AS; en los lactantes. Los microorganismos patógenos habituales son los estreptococos del grupo B, los bacilos intestinales Gram negativos y Staphylococcus aureus ( $S$. aureus). En niños menos de 5 años son: $S$. aureus, Streptococcus pyogenes y Kingella kingae ( $K$. kingae), mientras los adultos jóvenes y adolescentes, Neisseria gonorrhoeae ( $N$. gonorrhoeae) es el patógeno más frecuente (14).

Por otro lado, la presencia de gérmenes Gram negativos es más frecuente en pacientes con sistema inmunológico comprometido como: drogadictos, heridas contaminadas y tumores gastrointestinales, la incidencia por este tipo de microorganismo es de alrededor del $1 \%$ y se asocian por lo general a la presencia de la Chlamydia. Las infecciones secundarias a una cirugía o a lesiones penetrantes, se deben principalmente a $S$. aureus. Las infecciones por estafilococos coagulasa negativo son poco frecuente salvo después de artroscopia o de colocación de prótesis articular.

Tabla1. Factores de riesgo para la artritis séptica de la rodilla en adultos y niños

\begin{tabular}{ll|}
\hline \multicolumn{1}{c|}{ Niños } \\
- & Prematuridad \\
- & Síndrome de distrés respiratorio \\
\hline & \multicolumn{1}{c|}{ Adultos } \\
- & Enfermedades crónicas: diabetes mellitus, \\
- alcoholismo e insuficiencia renal \\
- Enfermedades articulares crónicas: artritis \\
- $\quad$ Antecedenatentes de trauma \\
- Cirugías previas, incluye artroscopia \\
- Estados de inmunosupresión \\
- Edad avanzada (mayores de 60 años) \\
- Uso y abuso de drogas intravenosas \\
\hline Fuente. Alejandro Álvarez López, Sergio Ricardo \\
Soto-Carrasco, Yenima de la Caridad García \\
Lorenzo. Septic arthritis of the knee.AMC vol.22 \\
no.4 Camagüey jul.-ago. 2018: $609-626$ \\
\hline
\end{tabular}

\section{CUADRO CLÍNICO}

El cuadro clínico inicial suele ser poco específico, como irritabilidad (especialmente en los niños más pequeños) y fiebre, normalmente no muy elevada y no siempre presente. El síntoma más característico y constante es el dolor, con postura antiálgica, rechazo de la movilización o impotencia funcional, acompañada de tumefacción y calor local.

El eritema no suele estar presente, salvo que se asocie a infecciones de partes blandas. En las articulaciones profundas, como la cadera, el calor y la tumefacción no son apreciables. En los recién nacidos, la clínica es totalmente inespecífica, pudiendo presentarse como una seudoparálisis de la articulación afectada o como un cuadro 
séptico. Las articulaciones más afectadas son: cadera $44 \%$, rodilla $28 \%$ y tobillo $18 \%$ y la localización poliarticular se encontró en el $5 \%(15,16)$.

Las complicaciones más frecuentes son: limitación para deambular, alteración del crecimiento óseo, afectación articular con movilidad limitada, que se describen en el $10-25 \%$ de los casos. El mayor riesgo de secuelas se asocia a retraso en el diagnóstico y tratamiento, afectación de lactantes pequeños e infección por Gram negativos o S. aureus (16).

\section{DIAGNÓSTICO}

Para el diagnóstico nos ayudarán, las pruebas de laboratorio como, el aumento de la velocidad de sedimentación globular (VSG) y de la proteína $C$ reactiva (PCR) es habituales, pero inespecífico, y su normalidad no descarta la infección. La leucocitosis moderada es igualmente habitual. Con evoluciones favorables, la PCR se normaliza entre 7 y 10 días, mientras que la VSG alrededor de 3-4 semanas. El aumento $o$ la persistencia de niveles elevados de la PCR es un dato indicativo de evolución desfavorable o de desarrollo de complicaciones, por encima, incluso de la presencia de fiebre (17).

La identificación del patógeno responsable es la confirmación de la etiología infecciosa y nos ayudará a instaurar el tratamiento más adecuado. Por ello, siempre es recomendable realizar toma de hemocultivos ya que al menos un tercio de los casos asocian bacteriemia. Asimismo, se debe obtener líquido articular para cultivo y, si es posible, análisis por medio de técnicas moleculares para la identificación del microorganismo causal. A pesar de todo, la identificación final del patógeno responsable se produce en un $50-80 \%$ de casos. Ante la sospecha clínica y con los laboratorios sugestivos de AS, la articulación infectada debería drenarse lo antes posible, por artrocentesis, artroscopia o artrotomía. En este caso, el inicio de la antibioterapia empírica, igualmente, debería demorarse hasta la obtención de líquido sinovial para estudio. La ecografía es la prueba de imagen de elección en el diagnóstico de la AS y puede guiar la artrocentesis. Identifica el derrame articular, si bien, no es capaz de discriminar entre una AS o inflamatoria $(15,18,19)$.

\section{Diagnóstico microbiológico}

Ante la sospecha de AS es muy importante la toma de muestras (hemocultivos y líquido articular) para buscar el diagnóstico etiológico que permita el tratamiento más adecuado. El líquido articular se analizará en fresco para ver el recuento celular y realización de Gram.

Estas dos técnicas dan una información provisional de máxima utilidad para la orientación terapéutica del paciente. Además, debe inyectarse en frascos de hemocultivos para facilitar el crecimiento de microorganismos de difícil aislamiento como $K$. kingae. Se podían realizar técnicas de biología molecular (16).

El análisis de química sinovial como la concentración de glucosa y proteínas no son de utilidad en el diagnóstico, al igual que los marcadores inflamatorios como el recuento de leucocitos, VSG y PCR tienen un valor limitado, ya que pueden presentarse en otras afecciones articulares (2,19). A diferencia de los niveles de procalcitonina sérica parece ser un marcador diferencial en la AS (19).

Basado en el análisis del líquido sinovial, los pacientes deben de ser tratados con un recuento de glóbulos blancos mayor a $50000 / \mathrm{mm}^{3}$. Sin embargo, un recuento menor no excluye el diagnóstico de AS $(2,20)$. En un estudio un $50 \%$ de los pacientes tenían un recuento de leucocitos menor a 50 mil. Los pacientes 
inmunosupresos pueden carecer de leucocitosis en el líquido sinovial (20).

\section{Técnicas de Imágenes}

El diagnóstico no se debe basar en hallazgos radiológicos, pero si pueden contar como un apoyo en la valoración de su evolución, en las primeras fases puede presentarse osteopenia articular o tumefacción de partes blandas, en la tercera semana se puede captar una disminución del espacio articular y posteriormente lesiones erosivas por osteítis subcondral que puede evolucionar a la destrucción epifisiaria (14).

\section{- Radiografía simple}

Es la primera técnica que se debería realizar, se evalúa signos indirectos de infección articular (aumento de tejidos blandos, desplazamiento de las estructuras musculares, aumento del espacio articular, luxación de la cadera), que orientan el diagnóstico. Permiten excluir otros posibles diagnósticos como traumatismos, enfermedades malignas, u osteomielitis (16).
- Ecografía

Es la técnica de elección con alta sensibilidad, pero baja especificidad para el diagnóstico de AS. Detecta derrame articular en fases muy iniciales, pero la técnica no permite diferenciar entre una artritis infecciosa 0 no infecciosa. En cambio, la ausencia de derrame articular puede casi excluir que el paciente tenga una AS. También puede identificar la presencia de derrame articular en articulaciones profundas, así como servir de guía para realizar la artrocentesis $(2,16)$.

\section{- Gammagrafía, TC, RMN}

Son técnicas que aportan poco y deben reservarse para casos especiales o de difícil diagnóstico, ya que las anteriores permiten diagnosticar la mayor parte de las artritis agudas. En casos donde se dificulte la obtención de líquido sinovial por punción se puede considerar realizar una gammagrafía osea, el trazador puede identificar la infección en las primeras 48 horas $(2,16)$.

\begin{tabular}{|l|l|l|l|l|}
\hline Tabla 2. Características del líquido articular \\
\hline Líquido articular & \multicolumn{1}{|c|}{ Normal } & \multicolumn{1}{|c|}{ Séptico } & \multicolumn{1}{|c|}{ Inflamatorio } & Traumático \\
\hline Color & Claro & Turbio & $+/-$ & $+/-$ \\
\hline Leucocitos/mm3 & $<200$ & $>50.000$ & $20.000-50.000$ & $10-25.000$ \\
\hline Neutrófilos \% & $<25$ & $>90$ & $50-80$ & $10-30$ \\
\hline $\begin{array}{l}\text { Glucosa mg/ml } \\
\text { Formación de } \\
\text { coágulo }\end{array}$ & $80-100$ & $<20$ & $20-50$ & $>50$ \\
\hline
\end{tabular}

Fuente. Hernández Sampelayo Matos T, Zarzoso Fernández S, Navarro Gómez ML. Santos Sebastián MM, González Martínez F, Saavedra Lozano J. Osteomielitis y artritis séptica. En: Asociación Española de Pediatría. Protocolos diagnóstico-terapéuticos de la AEP: Infectología pediátrica [Internet]. Madrid: AEP; 2008. p. 205-20. 


\section{DIAGNÓSTICO DIFERENCIAL}

La causa más frecuente de dolor de cadera en los niños entre 5 y 10 años es la sinovitis transitoria de cadera. Se presenta en niños con buen estado general, antecedente de infección respiratoria reciente, febrícula, dolor unilateral y limitación para deambular. Evoluciona a la curación tras unos días de reposo y antiinflamatorios. Es difícil de diferenciar de la fase inicial de una AS; varios autores se basan en scores para el diagnóstico. Un niño con dolor de cadera, fiebre, rechazo para caminar, VSG superior a $40 \mathrm{~mm} \mathrm{Hg}$ y leucocitos > 12.000 tienen gran probabilidad (60-99\%) de padecer una AS.

Existen artritis reactivas a distancia secundarias a una infección bacteriana previa. Estos procesos se producen por una reacción inflamatoria reactiva y no se aíslan microorganismos en la articulación. Es secundario a infecciones gastrointestinales por: Salmonella, Shigella, Campylobacter y Yersinia enterocolitica; infecciones genitourinarias por Chlamydia trachomatis o $\mathrm{N}$. gonorrhoeae; y otras infecciones producidas por Neisseria meningitidis y Streptococcus pyogenes. Es importante establecer el diagnóstico diferencial entre AS y otros procesos como traumatismos, osteomielitis, artritis reumatoide juvenil, enfermedades malignas y necrosis avascular idiopática de la cabeza femoral (enfermedad de LeggCalvé-Perthes) (16).

\section{TRATAMIENTO}

Se recomienda posponer la administración de tratamiento antibiótico posterior a la toma del cultivo de líquido sinovial debido a que son de gran utilidad, pero difieren en cuanto a la sensibilidad, ya que entre 40 y $50 \%$ resultan ser Gram negativa, mayor al $70 \%$ Gram positiva y menos del $25 \%$ de los casos corresponde a AS gonocócica $(2,19,20)$.
Está indicada la terapia antibiótica de amplio espectro, hasta obtener el resultado del cultivo de líquido sinovial, esto por su potencial veloz destructivo (19).

\section{- Cocos Gram positivo}

Cloxacilina $2 \mathrm{~g} / 4$ horas vía intravenosa (IV) o cefazolina 2 g cada 8 horas IV $(2,19)$.

En los casos con factor de riesgo de infección por $S$. aureus meticilino resistente (SAMR) se puede iniciar cobertura con vancomicina, cuya dosis 1 gr cada 12 horas IV, así como linezolid $600 \mathrm{mg}$ cada 12 horas IV o vía oral (VO) ya que cuenta con una buena disponibilidad, también se puede contar con daptomicina 6-10 $\mathrm{mg} / \mathrm{kg} /$ día IV $(2,19)$.

\section{- Bacilos Gram negativos}

Pacientes sexualmente activos, asociada a una clínica de infección gonocócica diseminada debe de recibir ceftriaxona $1 \mathrm{gr}$ cada 12 horas IV o IM. También es aceptable usar cefotaxima 2 g cada 8 horas IV asociado o no a amikacina $15 \mathrm{mg} / \mathrm{kg} /$ día IV en los primeros 3 a 5 días (2).

Al confirmar el diagnóstico por $\mathrm{N}$. gonorrhoeae, se puede continuar vía oral con cefixima $200 \mathrm{mg}$ cada 12 horas o en caso de ser sensible se puede utilizar ciprofloxacina $750 \mathrm{mg}$ cada 12 horas (2). En el caso de pacientes con hipersensibilidad a betalactámicos puede utilizarse aztreonam 1-2 g cada 8 horas IV o ciprofloxacino 400 mg cada 812 horas IV o $750 \mathrm{mg}$ cada 12 horas VO (2).

Cefepima o un betalactámico antipseudomónico, en el caso de pacientes gravemente enfermos, como ancianos, inmunodeprimidos o usuarios de drogas intravenosas. Así como el uso de un carbapenem como meropenem 2 gr cada 8 horas IV $(2,19)$. 


\section{- Mordeduras de animales o herida penetrante sucia}

Amoxicilina - clavulánico 2-0,2 gr cada 8

horas IV, piperacilina - tazobactam 4-0,5 gramos cada 6-8 horas IV o la asociación de cefotaxima 2 gramos cada 8 horas o cefriaxona $1 \mathrm{gr}$ cada 12-24 horas IV con metronidazol.

En cuanto a la duración del tratamiento, en adultos, no gonocócica debe de ser de al menos 2-4 semanas, seguido terapia oral escalonada por 4 semanas. En población infantil, una AS no complicada con 10 días de cobertura es suficiente. Infección gonocócica puede tratarse 2 semanas con ceftriaxona. En la afectación de articulaciones esternoclavicular y sacroiliaca, la cobertura debe de ser por 6 semanas, ya que en esta localización es común su complicación con osteomielitis (19).

\section{Drenaje articular}

La terapia antibiótica más el drenaje articular, es la combinación realizada para el abordaje de AS. Su función es descomprimir la articulación, eliminar toxinas y favorecer el flujo sanguíneo lo que ayudaría a una mejor distribución del antibiótico (2).

El drenaje se puede realizar mediante artroscopía, artrocentesis o artrotomía preferiblemente la artrocentesis y así por medio de esta evaluar evolución del cuadro mediante el análisis del líquido sinovial que se vaya extrayendo $(2,19)$.

Los casos de AS de cadera, inadecuada evolución clínica, cultivo positivo a los 5 días y acúmulo de fibrina 0 adherencias que formen tabiques en el espacio articular se debe valorar la realización de un drenaje quirúrgico y desbridamiento (2).

La rehabilitación precoz es importante para evitar contracturas y atrofia muscular además en estudios recientes se ha identificado que con la inmovilización articular es mayor el riesgo de degeneración del cartílago y formación de adherencias $(2,19)$.

\section{CONCLUSIONES}

La AS es una afección de la articulación que puede provocar secuelas funcionales importantes. La forma de presentación es por lo general aguda con la presencia de síntomas y signos locales, así como generales. El germen con más participación en esta enfermedad es el $S$. aureus, de allí que la terapia antimicrobiana empírica debe estar encaminada contra este microorganismo.

Ante la sospecha debe de solicitarse una analítica sanguínea y pruebas de imagen, como ecografía o una radiografía simple de la articulación afectada. En caso de no tener una claridad del cuadro se debe de realizar una artrocentesis y cultivo del líquido articular, con el fin de obtener la mejor opción terapéutica (20).

El manejo de la artritis debe ser multidisciplinario con la participación de cirujanos ortopédicos que puedan hacer la punción diagnóstica y tratamiento quirúrgico precozmente, pediatras, infectólogos y rehabilitadores implicados en el tratamiento médico, seguimiento y rehabilitación de estos pacientes (16).

\section{Los autores declaran no tener conflicto} de interés.

\section{REFERENCIAS}

1. Vigorita VJ. Orthopaedic pathology. 3 er ed. Philadelphia: Wolters Kluwer; 2016. p. 281-7.

2. Múñez Rubio E, Pintos Pascual I, Ramos Martínez A. Artritis infecciosa. Medicine - Programa de Formación Médica Continuada Acreditado. 2018;12(55):3253-3261

3. Prior-Español Á, García-Mira $Y$, Mínguez $S$, Martínez-Morillo M, Gifre L, Mateo L. Coexistencia de AS y microcristalina: un reto diagnóstico. A propósito de 25 casos. Reumatología Clínica. 2019;15(6):e81-e85 
4. Borri Á, Bazán P, Medina M, Maximiliano Ciccioli N. AS facetaria en columna. Ortho-tips. 2021;17(1):6-10

5. Yeo A, Ramachandran M. Acute haematogenous osteomyelitis in children. BMJ [Internet]. 2014 [cited 2018 May 20]; 348: g66-g66. Available from: http://www.bmi.com/cgi/doi/10.1136/bmi.g66

6. Begly JP, Sobieraj M, Liporace FA, Dayan A. Staphylococcus lugdunensis septic arthritis of a native knee a case report. Bull Hosp Jt Dis. 2016 Nov;74(4):314-7.

7. Bono KT, Samora JB, Klingele KE. Septic arthritis in infants younger than 3 months: a retrospective review. Orthopedics. 2015 Sep;38(9):e787-93

8. Mascioli AA, Park AL. Infectious arthritis. En: Azar FM, Beaty JH, Canale ST, editors. Campbell\&apos;s Operative Orthopaedics. 13 th ed. Philadelphia: Elsevier; 2017. p. 793-6

9. Carlos Antonio Guillen, Marta Grandal, Carmen Velazquez, Valentina Luidmila, Mónica Vásquez. Guía de Manejo diagnóstico y terapéutico de la AS en urgencias. Vol 9 No .2:3. 2013: 1-10.7.0 C. Calvo Rey y J. Saavedra-Lozano, El tratamiento de la AS ,An Pediatr Contin. 2014;12(6):325-9

10. Alejandro Álvarez López, Sergio Ricardo SotoCarrasco, Yenima de la Caridad García Lorenzo. Septic arthritis of the knee.AMC vol.22 no.4 Camagüey jul.-ago. 2018: 609-626

11. Markus Pääkkönen, and Heikki Peltola. Revisión del tratamiento de la AS aguda en la infancia. 2013.

https://www.intramed.net/contenidover.asp?conte nidoid $=81030$

12. N. Gómez Rodríguez, J. Ibáñez Ruán, $M$. González, A. Pintado, Y. Penelas Cortés. ASs periféricas en adultos. Vol. 18, N. ${ }^{\circ} 11$ 2001: 573577

13. David Figueroa, Francisco Figueroa, Marilaura Núñez, Vicente Monte. Estrategias para minimizar el riesgo de infección posterior a una reconstrucción de ligamento cruzado anterior. Artroscopia. Vol. 28 Núm. 1 (2021):92-96

14. Jameson, Fauci, Kasper, Hauser, Longo, Loscalzo. Harrison Principios de Medicina Interna $20^{a}$ edición. McGraw-Hill Education 2018: 939940

15. S. Atehortúa, et al. Diagnóstico microbiológico de AS en niños usando botellas de hemocultivos como un método alternativo. Infectio 2020; 24(2):98-102. https://doi.org/10.22354/in.v24i2.840

16. Hernández Sampelayo Matos $T$, Zarzoso Fernández S, Navarro Gómez ML. Santos Sebastián MM, González Martínez F, Saavedra Lozano J. Osteomielitis y AS. En: Asociación Española de Pediatría. Protocolos diagnósticoterapéuticos de la AEP: Infectología pediátrica [Internet]. Madrid: AEP; 2008. p. 205-20. [citado
19 Mar 2016]. Disponible en: http://www.aeped.es/sites/default/files/documento s/osteomielitis.pdf

17. S.N. Faust, J. Clark, A. Pallett, N.M. Clarke. Managing bone and joint infection in children. Arch Dis Child., 97 (2012), pp. 545-553 http://dx.doi.org/10.1136/archdischild-2011301089

18. Paakkonen M. Septic Arthritis in children:diagnosis and treatment. Pediatric Health Med Ther. 2017;8:65-68

19. Ross J. Septic Arthritis of Native Joints. Infectious Disease Clinics of North America. 2017;31(2):203218.

20. Moro-Lago I, Talavera G, Moraleda L, GonzálezMorán G. Presentación clínica y tratamiento de las ASs en niños. Revista Española de Cirugía Ortopédica y Traumatología. 2017;61(3):170-175. 